\title{
INCERTIDUMBRE TAXONÓMICA DE UN PRIMATE NEOTROPICAL; EL CASO DE Ateles fusciceps rufiventris SCLATER, 1871 (PRIMATES: ATELIDAE).
}

\author{
Juan C. Cepeda-Duque ${ }^{1}$, Andrés Link ${ }^{2}$ y Hugo Mantilla-Meluk ${ }^{3}$ \\ ${ }^{1}$ Universidad de Santa Rosa de Cabal, Facultad de Ciencias Básicas, Programa de Biología, Grupo de Investigación en \\ Biología de la Conservación y Biotecnología, Santa Rosa de Cabal, Risaralda, Colombia. \\ [Correspondencia: Juan C. Cepeda-Duque < acinonyxjubatus96@gmail.com>] \\ ${ }^{2}$ Departamento de Ciencias Biológicas y Facultad de Administración, Universidad de los Andes. \\ ${ }^{3}$ Programa de Biología, Universidad del Quindío, Armenia, Colombia.
}

RESUMEN. La taxonomía de los monos araña (Ateles spp.) en el Pacífico ha sido un tema de controversia en la literatura científica, con varias hipótesis derivadas de análisis biogeográficos, morfológicos y genéticos, tendiendo a volver difusa la certidumbre concerniente a la identidad específica de este taxón, lo cual complejiza la toma de decisiones para la conservación de este grupo de primates en el Neotrópico. Por lo tanto, en este estudio presentamos una revisión sistemática que soporta que las poblaciones de mono araña de la porción norandina del Pacífico deberían atribuirse a Ateles fusciceps rufiventris.

ABSTRACT. Taxonomic uncertainty in a Neotropical primate: the case of Ateles fusciceps rufiventris Sclater, 1871 (Primates: Atelidae). The taxonomy of spider monkeys (Ateles spp.) in the Colombian Pacific region has been controversial in the scientific literature, with several hypotheses derived from biogeographical, morphological and genetic analyzes. Uncertainty concerning the specific identity of this taxon makes decision making more difficult for the conservation of this group of primates in the Neotropics. In this study, we present a systematic review which supports that populations of spider monkeys of the north-Andean portion of the Pacific should be assigned to Ateles fusciceps rufiventris.

Palabras clave: Ateles fusciceps rufiventris, conservación, taxonomía, Región Pacífica, Risaralda.

Key words: Ateles fusciceps rufiventris, conservation, taxonomy, Pacific region, Risaralda.

Los monos araña (género Ateles) son unos de los mamíferos neotropicales con mayor vulnerabilidad a la degradación y fragmentación de su hábitat, así como a la cacería (Michalski \& Peres 2005). De acuerdo a lo anterior, todas las especies de este género han sido categorizadas en estado de peligro (EN) o peligro crítico (CR) y se encuentran entre las principales prioridades de conservación (Stevenson et al. 2010). Las poblaciones de A. fusciceps en el noroccidente de Sur América están amenazadas por extinción a corto o mediano plazo debido a la reducción sistemática de sus poblaciones como producto de la cacería indiscriminada y la alarmante tasa 
de trasformación de los ambientes naturales, en particular de áreas con cobertura boscosa como producto de la ampliación de la frontera agrícola y ganadera y de la minería (Defler \& Bueno 2010). El estatus taxonómico y los límites de la distribución geográfica de los monos araña negros centroamericanos y del norte del subcontinente suramericano han sido temas de debate (Ruíz-García et al. 2016). En este trabajo, presentamos una semblanza cronológica sobre la taxonomía de los monos araña negros del norccidente de Sur América.

La propuesta taxonómica aceptada en la actualidad considera a $A$. $f$. rufiventris como el nombre aplicable a las poblaciones de mono araña del occidente de Colombia. Las poblaciones de mono araña del occidente de Colombia se caracterizan morfológicamente por ser ejemplares con una menor dimensión de las extremidades, mayor robustez corporal, cola ligeramente más corta y relativamente menos esponjosa (Kellogg \& Goldman 1944). Además, su cráneo es más ancho que el de las formas típicas de Centroamérica y la vertiente oriental de los Andes (Tabla 1), apariencia que se resume en el epíteto robustus utilizado por Allen (1914). Finalmente, otros caracteres que identifican a los individuos del género Ateles del occidente colombiano son su pelaje negro grueso y brillante (Allen 1914), un rostro completamente negro, excepto por algunas vibrisas y pelos blancos dispersos en la quijada y, en ocasiones, un bigote o bozo blanco.

Los monos araña del occidente colombiano se cuentan entre los mamíferos menos conocidos en el país y este desconocimiento se ve reflejado en la falta de claridad con relación a sus relaciones filogenéticas e identidad taxonómica de sus poblaciones (Kellogg \& Goldman 1944; Hershkovitz 1969, 1972, 1977; Heltne \& Kunkel 1975; Hernández-Camacho \& Cooper 1976; Kunkel et al. 1980; Wolfheim 1983; Hernández \& Defler 1986, 1989; Froehlich et al. 1991; Medeiros et al. 1997; Collins \& Dubach 2000 a; b, 2001; Groves 2001; Ruiz-García \& Álvarez 2003; Defler 2004, 2010; Nieves et al. 2005; Ruíz-García et al. 2006, 2016; Rylands et al. 2006; Morales-Jiménez 2007; Morales-Jiménez et al. 2015). El nombre rufiventris, Sclater 1871 (datado erróneamente en la UICN como 1872), está asociado a la descripción de un ejemplar juvenil, con localidad tipo en río Atrato, Darién, en el Chocó biogeográfico colombiano, y es el único que se asocia directamente a poblaciones del género Ateles provenientes de la vertiente occidental de la cordillera Occidental.

Parte de las dificultades en su designación taxonómica se relaciona al uso común del nombre robustus, asociado a la descripción de Allen (1914), la cual se realizó a partir de cuatro ejemplares provenientes de la vertiente oriental de la cordillera Occidental, en la localidad La Gallera, municipio de El Tambo, departamento de Cauca (Allen 1914) (AMNH 32352-55 ठ̊), holotipo colectado por L. E. Miller, el 11 de julio de 1911 y un ejemplar proveniente de Barbacoas, departamento de Nariño, en la vertiente Pacífica, de la cordillera Occidental (AMNH 34155 ${ }^{\AA}$ ); todos preservados como pieles y cráneos (Fig. 1).

Basados principalmente en la variación de color del pelaje, Kellogg \& Goldman (1944), en su trabajo seminal, propusieron cuatro especies del género Ateles: A. belzebuth, A. geoffroyi, A. paniscus y A. fusciceps, siendo esta última la forma reconocida para la vertiente occidental de los Andes en Panamá, Colombia y Ecuador (Defler 2010). Kellogg \& Goldman (1944) asignaron para Colombia dos subespecies: 1) A. f. robustus, como la forma distribuida a lo largo de las tierras bajas del Urabá antioqueño, la costa atlántica en los departamentos de Córdoba, Sucre y el norte de Bolívar; extendiendo su distribución al oriente hasta el bajo río Cauca sobre su ribera occidental y a la porción central de Antioquia y todo el Pacífico colombiano al occidente; y 2) A.f. grisescens Gray 1866 con localidad tipo en el río Tuyra, sureste de Panamá (espécimen depositado en el Museo Británico de Historia Natural, $\mathrm{N}^{\circ} 1865.4 .20 .2$; Napier 1976) presente también en las estribaciones de la Serranía del Baudó en el departamento del Chocó (Defler 2010).

Por su parte, Hershkovitz (1969, 1972, 1977), Hernández \& Cooper (1976) y Wolfheim (1983) reconocieron para el territorio colombiano una única especie, A. paniscus, para la cual señalaron una alta variabilidad con cuatro (Hernández \& Cooper 1976) o 
Tabla 1

Revisión bibliográfica del estado taxonómico de Ateles fusciceps rufiventris.

\begin{tabular}{|c|c|c|}
\hline Autor & Nombre científico & Evidencia \\
\hline Allen (1914) & robustus & Morfología \\
\hline Kellogg \& Goldman (1944) & fusciceps & Morfología \\
\hline Hershkovitz $(1969,1972,1977)$ & A. paniscus (incluyendo fusciceps y geoffroyi) & Morfología \\
\hline Heltne \& Kunkel (1975) & A fusciceps rufiventris & Morfología \\
\hline Hernández \& Cooper (1976), & paniscus robustus & Morfología \\
\hline Kunkel et al. (1980) & A. f. rufiventris & Cariológica \\
\hline Wolfheim (1983) & paniscus robustus & Morfología \\
\hline Hernández \& Defler $(1986,1989)$ & A. p. robustus & Morfología \\
\hline Froehlich et al. (1991) & A. geoffroyi & Morfología \\
\hline Medeiros et al. (1997) & A. $f$. rufiventris & Cariológica \\
\hline Groves (2001) & A. fusciceps rufiventris & Revisión bibliográfica \\
\hline Collins \& Dubach (2000 a,b, 2001) & A. geoffroyi robustus & Molecular \\
\hline Ruiz-García \& Alvarez (2003) & A. $f$. robustus y A. f. rufiventris & Molecular \\
\hline Defler (2004) & A. g. rufiventris & Revisión bibliográfica \\
\hline Nieves et al. (2005) & A. g. rufiventris & Cariológica y Molecular \\
\hline Rylands (2006) & A. $f$. rufiventris & Revisión bibliográfica \\
\hline Ruíz-García et al. (2006) & A. f. robustus & Molecular \\
\hline Morales-Jiménez (2007) & A. fusciceps & Revisión bibliográfica \\
\hline Defler (2010) & A. g. rufiventris & Revisión bibliográfica \\
\hline Morales-Jiménez et al. (2015) & A. fusciceps & Molecular \\
\hline Ruíz-García et al. (2016) & A. f. rufiventris & Molecular \\
\hline
\end{tabular}

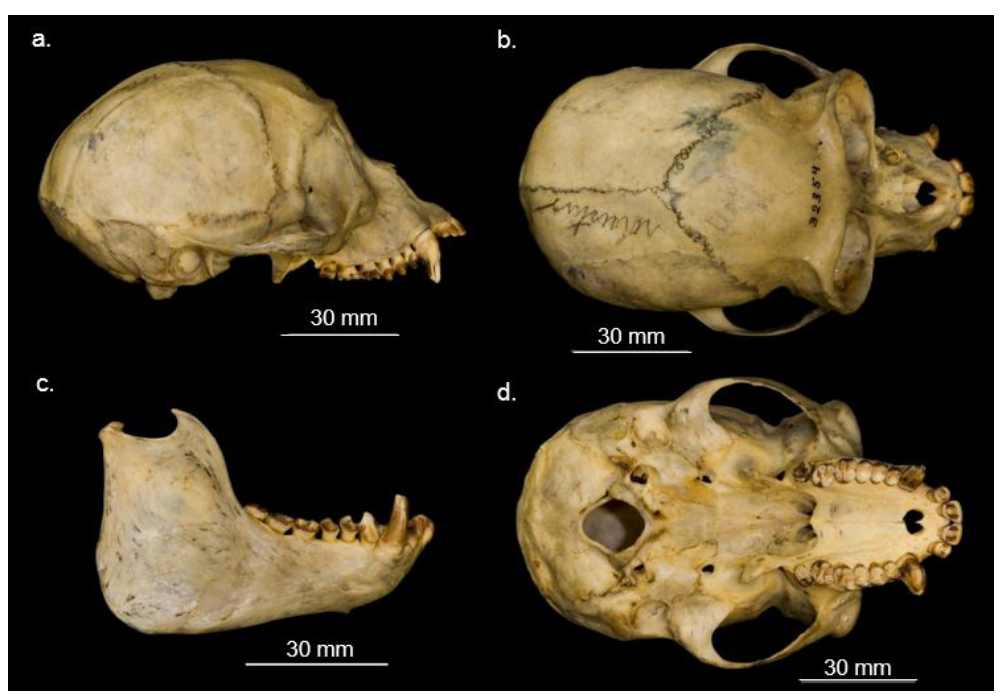

Fig. 1. Holotipo de Ateles robustus (Allen 1914) AMNH32354. a) Vista latera del maxilar superior, b) vista dorsal del maxilar superior, c) vista lateral del maxilar inferior y d) vista ventral del maxilar superior. Créditos: American Museum of Natural History. 
cinco (Hernández \& Defler 1986, 1989) subespecies, estando A. p. fusciceps restringida al extremo noroccidental de Colombia en límites con Panamá y siendo A. p. robustus la forma reconocida para la región pacífica (Defler 2010). Más recientemente, se ha reconocido que el grupo paniscus corresponde a una subespecie de los monos araña de la Amazonía occidental distribuidos en Perú y Brasil (Collins \& Dubach 2000a; Morales-Jiménez et al. 2015), asignación probablemente asociada al color negro de su pelaje.

Basados en evidencia cariológica, Kunkel et al. (1980), al igual que Medeiros et al. (1997), reconocen a $A$. $f$. rufiventris como la forma correspondiente al occidente de Colombia en la región pacífica, diferente de $A$. $f$. fusciceps del occidente de Ecuador.

Más adelante, Collins \& Dubach (2000 a; b), en un análisis filogenético basado en secuencias de ADN mitocondrial, establecen que todas las subespecies del primate centroamericano A. geoffroyi forman un único clado con las formas de A. fusciceps, indicando la ausencia de barreras físicas recientes entre las poblaciones de Ateles del sur de Centroamérica y aquellas de la vertiente occidental de la porción norte de América del Sur. De hecho, estos autores mencionan la presencia de híbridos en estado silvestre entre clados centro y suramericanos. Collins \& Dubach (2000 a) reconocen a la subespecie robustus como la forma propia de la biorregión colombiana del Chocó-Magdalena, y fusciceps como la subespecie típica de la vertiente pacífica del territorio ecuatoriano.

Por su parte, Ruiz-García \& Álvarez (2003), concluyen de sus análisis filogenéticos, basados en datos derivados de secuencias de ADN mitocondrial y RFLPs que $A$. f. robustus y A.f. rufiventris no son necesariamente sinónimos, sugiriendo una distribución de A. f. robustus en el Chocó y otras áreas del Pacífico, al tiempo que A.f. rufiventris es la forma correspondiente al Alto Sinú y el departamento de Córdoba.

La revisión del género Ateles de Raylands et al. (2006) sigue la propuesta taxonómica de Kellogg \& Goldman (1944) asignando a las poblaciones centroamericanas el epíteto geoffroyi y reservando el epíteto fusciceps para las poblaciones suramericanas.

Ruiz-García et al. (2006), basados en análisis moleculares de microsatélites de DNA, que incluyeron muestras de Turbo, Antioquia; Colosó Sucre; Barranquilla, Atlántico; Alto Sinú, Córdoba; Darién, Parque Nacional Los Katios, y Riosucio, Chocó, establecen a A. f. robustus como la forma del género distribuida en el Pacífico colombiano, que se reemplaza al sur de su distribución, en los límites de la frontera colombo-ecuatoriana, por la subespecie fusciceps (Tirira et al. 2011); esta ha sido sugerida para el territorio colombiano, aunque no existen registros para el país a la fecha en la literatura científica.

Por su parte, Froehlich et al. (1991), basados en el análisis de 50 caracteres craneales, retoman la visión de Kellogg \& Goldman (1944) y sugieren a $A$. fusciceps como especie completa independiente de su forma congenérica A. geoffroyi en Centro América; interpretación compartida por la evidencia cariológica en Medeiros et al. (1997) y más recientemente por los trabajos basados en datos moleculares de Groves (2001). Un trabajo reciente realizado por Morales-Jiménez et al. (2015) comparando secuencias de ADN mitocontrial da soporte al estatus específico de estos dos taxones y estima que su tiempo de divergencia data aproximadamente de 2.2 Mya. Así mismo, los estudios de Morales-Jiménez, sugieren una subdivisión dentro de A. fusciceps, ya que poblaciones en el norte de su distribución (Panamá) y en el sur de su distribución (Ecuador) compartieron un ancestro común hace aproximadamente 1.4 Mya. Finalmente, Ruíz-García et al. (2016), basados en datos moleculares de los genes mitocondriales COI, COII y cyt-b de 283 individuos de Ateles, que incluyen 89 especímenes de A.f.rufiventris de Colombia (Antioquia, Atlántico, Cauca, Córdoba, Chocó, Nariño, Sucre y Valle del Cauca) y cinco ejemplares de A. f. fusciceps de la región pacífica de Ecuador, más siete formas Centroamericanas de geoffroyi (incluyendo muestras de A. g. griscecens del Chocó), determinan que las poblaciones de A. fusciceps de Colombia y Ecuador son indiferenciables desde la perspectiva molecular. 
Lo anterior plantea la necesidad de análisis poblacionales más detallados para los monos araña distribuidos al occidente de los Andes que integren datos morfológicos y genes nucleares, que permitan una mayor resolución filogenética.

En conclusión, hay una congruencia en los resultados de la mayor parte de los estudios sobre las relaciones filogenéticas de los monos araña del occidente de Los Andes. El primer resultado que es consistente en casi todos los estudios basados en morfología, cariotipos y caracteres moleculares es el de indicar que las poblaciones de monos araña en el Pacífico de Colombia y Ecuador forman un grupo monofilético, que corresponde al grupo hermano de los monos araña de Centro América (A. geoffroyi). El segundo resultado consistente en varios estudios es la subdivisión taxonómica de las poblaciones de A. fusciceps en dos grupos principales: el primero, A. f. rufiventris, se encuentra en la zona norte de la distribución de la especie, desde el sur de Colombia (en Nariño y Cauca) hasta el Chocó biogeográfico y los bosques del Caribe en Colombia, y el segundo, A. f. fusciceps, está restringido a los bosques del occidente de Ecuador.

Agradecimientos. Agradecemos al American Museum of Natural History por poner a disposición del público en general el material fotográfico del holotipo, al Semillero Interinstitucional de Estudio y Conservación de los Carnívoros del Eje Cafetero y al Centro de Estudios de Alta Montaña.

\section{LITERATURA CITADA}

Allen, J. A. 1914. New South American monkeys. Bulletin of American Museum of Natural History 33:647-655.

Collins, A. C., \& J. M. Dubach. 2000a. Phylogenetic relationships of spider monkeys (Ateles) based on mitochondrial DNA variation. International Journal of Primatology 21:381-420.

Collins, A. C., \& J. M. Dubach. 2000b. Biogeographic and ecological forces responsible for speciation in Ateles. International Journal of Primatology 21:421-444.

Collins, A. C., \& J. M. Dubach. 2001. Nuclear DNA variation in spider monkeys (Ateles). Molecular Phylogenetics and Evolution 19:67-75.

Defler, T. R. 2004. Primates of Colombia. Serie de Guías Tropicales de Colombia 4. Conservación Internacional, Bogotá D.C.

Defler, T. R. 2010. Historia natural de los primates colombianos. Universidad Nacional de Colombia.
Facultad de Ciencias. Departamento de Biología. Bogota D. C.

Defler, T. R., \& M. L. Bueno. 2010. Prioridades en investigación y conservación de primates colombianos. Primatología en Colombia: avances al principio del milenio, Primera Edición, Bogotá D. C. 193-214.

Froehlich, J. W., J. Supriatna, \& P. H. Froehlich. 1991. Morphometric analysis of Ateles: Systematics and biogeographic implications. American Journal of Primatology 25:1-22.

Groves, C. P. 2001. Primate taxonomy. Washington, D. C.: Smithsonian Institution Press.

Heltne, P. G, \& L. M. Kunkel. 1975. Taxonomic notes on the pelage of Ateles paniscus paniscus, A. p. chamek (sensu Kellogg and Goldman, 1944) and A. fusciceps rufiventris ( $=$ A. f. robustus, Kellogg and Goldman 1944). Journal of Medical Primatology 4:83-102.

Hernández-Camacho, J., \& R. W. Cooper. 1976. The nonhuman primates of Colombia. Neotropical Primates: Field Studies and Conservation (R. W. Thorington Jr. \& P. G. Heltne, eds.). National Academy of Sciences, Washington D. C.

Hernández-Camacho, J., \& T. R. Defler. 1986. Some aspects of the conservation of non-human primates in Colombia. Primate Conservation 6:42-50.

Hernández-Camacho, J., \& T. R. Defler. 1989. Algunos aspectos de la conservación de primates no-humanos en Colombia. La Primatología en Latinoamérica. (C. J. Saavedra, R. A. Mittermeier \& I. Bastos-Santos, eds.). WWF-U.S, Washington, D. C.

Hershkovitz, P. 1969. The evolution of mammals in southern continents. VI. The recent mammals of the Neotropical region: a zoogeographic and ecological review. Quarterly Review of Biology 44:1-70.

Hershoovitz, P. 1972. The recent mammals of the Neotropical region: a zoogeographic and ecological review. Evolution, mammals and southern continents. (A. F. Keast, C. Erk \& B. Glass, eds.). Albany, State University of New York.

Hershkovitz, P. 1977. Living New World monkeys (Platyrrhini). Volumen 1, University of Chicago Press, Chicago.

KellogG, R., \& E. A. Goldman. 1944. Review of the spider monkeys. Proceedings of the United States Natural Museum 96:1-45.

Kunkel, L. M., P. G. Heltne, \& D. S. Borgaonkar. 1980. Chromosomal variation and zoogeography in Ateles. International Journal of Primatology 1:223-232.

Medeiros, M. A. ET AL. 1997. Radiation and speciation of spider monkeys, genus Ateles, from cytogenetic viewpoint. American Journal of Primatology 42:167-178.

Michalski, F., \& C. A. Peres. 2005. Anthropogenic determinants of primate and carnivore local extinctions in a fragmented forest landscape of southern Amazonia. Biological conservation 124:383-396.

Morales-Jiménez, A. 2007. Action Plan of the spider monkeys Ateles hybridus and Ateles fusciceps in Colombia. <http://www.fundacionbiodiversa.org/pdf/ Ateles/Atelesactionplanweb.pdf $>$.

Morales-Jimenez, A. L., T. Disotell, \& A. Di Fiore. 2015. Revisiting the phylogenetic relationships, biogeography, 
and taxonomy of spider monkeys (genus Ateles) in light of new molecular data. Molecular phylogenetics and evolution 82:467-483.

NAPIER, P. 1976. Catalogue of primates in the British Museum (Natural History). British Museum of Natural History Publication. London.

Nieves, M., M. S. Ascunce, M. I. Rahn, \& M. D. Mudry. 2005. Phylogenetic relationships among some Ateles species: the use of chromosomic and molecular characters. Primates 46:155-164.

Ruiz-García, M., \& D. Alvarez. 2003. RFLP Analysis of mDNA from six platyrrhine genera: phylogenetic inferences. Folia Primatológica 74:59-70.

Ruiz-García, M., N. Lichilin, G. Gutierrez-Espeleta, M. I. Castillo, R. Wallace, \& P. Escobar-Armel. 2016. Molecular phylogeny of all the Ateles taxa (Atelidae, Primates) by means of mitochondrial genes and microsatellites. Molecular Phylogenetics and Evolution. Phylogeny, Molecular Population Genetics, Evolutionary Biology and Conservation of the Neotropical Primates (M. Ruíz-García \& J. M. Shostell, eds.). Nova Science Publisher Incorporations, New York.

Ruiz-García, M., A. Parra, N. Romero-Aleán, P. Escobar-Armel, \& J. M. Shostell. 2006. Genetic characterization and phylogenetic relationships between the Ateles species (Atelidae, Primates) by means of DNA microsatellite markers and craniometric data. Primate Report 73:3-47.
Rylands, A. B., C. P. Groves, R. A. Mittermeier, L. Cortés-Ortiz, \& J. J. Hines. 2006. Taxonomy and distributions of Mesoamerican primates. New perspectives in the study of Mesoamerican primates. (A. Estrada, P. A. Garber, M. M. Pavelka \& L. Luecke, eds.). Springer, United States.

Stevenson, P. R., D. C. Guzmán, \& T. R. Defler. 2010. Conservation of Colombian primates: an analysis of published research. Tropical Conservation Science, 3:45-62.

Tirira, D. G. ET AL. 2011. Mono araña de cabeza marrón (Ateles fusciceps). Libro Rojo de los mamíferos del Ecuador (D. G. Tirira, ed.), 2a . Edición. Fundación Mamíferos y Conservación, Pontificia Universidad Católica del Ecuador y Ministerio del Ambiente del Ecuador. Publicación especial sobre los mamíferos del Ecuador 8. Quito.

Wolfheim, J. H. 1983. Primates of the New World: Distribution, Abundance and Conservation. Seattle, University of Washington Press. 